\title{
Tumor-Associated Macrophage
}

National Cancer Institute

\section{Source}

National Cancer Institute. Tumor-Associated Macrophage. NCI Thesaurus. Code C116387.

Non-neoplastic macrophages that are found in close proximity to or within a tumor mass. 